\title{
LOS DERECHOS DEL NIÑO EN EL MARCO DE LA BIOETICA
}

\author{
CORONEL Aquino, Nancy ${ }^{75}$
}

SUMARIO: Valoración ética - Valoración jurídica - Conclusiones - Bibliografía

\section{RESUMEN}

El óvulo fecundado por un espermatozoide es ya persona humana, con un acto de ser, con un cuerpo y con un alma y por lo tanto su primer deseo humano es el derecho a vivir. El avance de la ciencia médico-biológica, incorpora nuevas situaciones fácticas que el derecho no puede dejar de comprender como es el caso de las consecuencias derivadas de la aplicación de las normas técnicas de reproducción humana asistida y específicamente a las técnicas de fertilización in vitro en los que el procedimiento utiliza espermatozoides y óvulos necesarios para producir sólo un embrión vivo pero varios embriones quedarían congelados sin saber cuál es su destino generando la pregunta, ¿qué sucede con aquellos seres humanos congelados en el laboratorio?. Este problema requiere de una legislación adecuada y urgente.

Palabras clave: Técnicas de reproducción humana asistida, técnicas de fertilización in vitro, derecho a la procreación, paternidad y maternidad, esterilidad e infertilidad, embriones crio conservados congelados, tráfico comercial, adopción pre-natal.

\begin{abstract}
Key words:

En estos últimos tiempos, con el avance de la ciencia médica - biológica se viene incorporando a nuestra vida diaria nuevas situaciones fácticas, que el derecho no puede quedar ajeno, nos referimos a las consecuencias de la aplicación de las nuevas Técnicas de Reproducción Humana Asistida ${ }^{76}$, y específicamente a las Técnicas de Fertilización In Vitro, en las que si bien se hace efectivo el derecho a la procreación y por ende el derecho a la paternidad y maternidad, como una solución de avance científico que ofrece a las parejas con problemas de esterilidad e infertilidad, sin embargo, es conocido que durante dicho procedimiento se utilizan no solo la cantidad de espermatozoides y óvulos necesarios para producir un solo embrión, sino varios, y consecuentemente como producto de dicho procedimiento, quedan embriones vivos sobrantes los mismos que se encuentran congelados en los laboratorios, sin saber cuál es su final; la pregunta que viene a colación es la siguiente:
\end{abstract}

\footnotetext{
${ }^{75}$ Juez Superior de Familia de la Corte Superior de Justicia de Lima.

${ }^{76}$ Definidas como métodos destinados a suplir la infertilidad en los seres humanos, logrando satisfacer el derecho a la procreación.
} 
¿Qué sucede con los otros embriones sobrantes?, ¿Qué sucede con aquellos seres humanos que permanecen congelados en los laboratorios reproductores, que no siendo utilizados para la reproducción de sus progenitores, se están acumulando en diferentes bancos de embriones?. Sin duda es un problema ético legal, que requiere de legislación urgente.

VALORACION ETICA. Cabe destacar, que en 1978, el Kennedy Institute de la Universidad Jesuita de Georgetown en Estados Unidos, publicó la primera Enciclopedia de Bioética dirigida por Warren Reich, un teólogo católico, donde se define a la Bioética como el "estudio sistemático de la conducta humana en el área de las ciencias de la vida y la salud, examinado a la luz de los valores y principios morales"; Este campo se dedica a encontrar una solución adecuada a los frecuentes dilemas éticos que se plantean en la práctica, en la investigación con seres humanos. Algunos consideran, como la iglesia católica, que debe prohibirse la investigación con seres humanos y por lo tanto no permitir la práctica clínica de la fecundación in vitro, sin embargo consideramos que ello no es una solución al problema, no obstante, resulta evidente la existencia de un número creciente de embriones crioconservados congelados, sin protección legal y susceptibles del tráfico comercial, como objetos de valor patrimonial, lo cual también constituye un problema ético y social relevante.

VALORACION JURIDICA. En principio se hace insoslayable, determinar ¿cuál es la naturaleza jurídica que tienen dichos embriones?, algunos consideran que tales embriones simplemente son un grupo de células susceptibles de ser utilizados en el laboratorio para la lucha de ciertas enfermedades, para detectar ciertas anomalías genéticas, o para mejoramiento de la raza humana, mientras que otros hacen distinciones otorgándoles un estatus jurídico de acuerdo al tiempo de procreación; que en efecto, en el caso de la legislación española, por ejemplo, el ser humano hasta los 14 días de concebido, le otorga la calidad "pre - embrión"; hasta los dos meses y medio, "embrión" y hasta su nacimiento, "feto". Entendiendo por "pre- embrión", "al embrión in vitro constituido por un grupo de células resultantes de la división progresiva del ovocito desde que es fecundado hasta 14 días más tarde", conforme lo regula el inciso 2 del Artículo $1^{\circ}$ de la Ley 14/2006, sobre "Técnicas de Reproducción Humana Asistida”.

Que en nuestro país, en el Código Civil, en su Artículo $1^{\circ}$ regula que "La persona humana es sujeto de derechos desde su nacimiento. La vida humana comienza con la concepción. El concebido es sujeto de derecho para todo cuanto le favorece. La atribución de derechos patrimoniales está condicionada a que nazca vivo.". Norma que implícitamente quedo derogada ${ }^{77}$ al entrar en vigencia el Código de los Niños y Adolescentes, (Decreto Ley 26102) con fecha 28 de junio de 1993, en el cual recogiéndose el "Sistema de Protección Integral" contenido en la Convención Sobre los Derechos del Niño ${ }^{78}$, en virtud del cual se definió "al niño" como persona humana desde la concepción, ello para evitar las manipulaciones o experimentos genéticos, puesto que el tratamiento condicionado a que nazca vivo, que le otorgó el Código Civil, implicaba también desprotegerlo de ciertos derechos;

Que en efecto, el Artículo I del Título Preliminar del Código de Los Niños y Adolescentes regula que "Se considera niño a todo ser humano desde su concepción hasta cumplir los doce años de edad.... El Estado protege al concebido para todo lo que le

${ }^{77}$ En merito a lo dispuesto en el Artículo I del Título Preliminar de dicho Código.

78 Adoptada por la Asamblea General de las Naciones Unidas en su resolución 44/25 del 20 de noviembre de 1989, y ratificada por el Perú mediante Resolución Legislativa número 25278 del 03 de agosto de 1990. 
favorece. ...". y el Artículo II del mismo cuerpo legal establece que "el niño es sujeto de derechos, libertades y de protección específica.".

Que en el quehacer jurisdiccional se conoció de un caso de Impugnación de Maternidad $^{79}$, en virtud del cual los padres biológicos hicieron realidad su derecho de tales, mediante fecundación in vitro, con la colaboración altruista de una tercera persona de sexo femenino, quien albergó y alimentó en su vientre durante toda la etapa de gestación a la niña concebida hasta su nacimiento; Que durante el curso de dicho proceso, se tomó conocimiento que durante el procedimiento de fertilización quedaron tres embriones congelados vivos los que se encontraban en un laboratorio clínico de ésta ciudad; Que ante tales circunstancias, y considerando a que conforme lo dispone el Artículo $1^{\circ}$ del citado Código de los Niños y Adolescentes, "El niño y el Adolescente tienen derecho a la vida desde el momento de la concepción." y que el citado Código de los Niños y Adolescentes "... garantiza la vida del concebido, protegiéndolo de experimentos o manipulaciones genéticas contrarias a su integridad y a su desarrollo físico o mental.", se tomaron las medidas necesarias a efectos de hacer efectivos los derechos fundamentales de los citados seres humanos crio conservados, como el derecho a la vida, derecho a crecer, derecho a nacer, derecho a conocer a sus padres, derecho a desarrollarse en el seno de una familia, etc. Se legislo en el caso concreto, toda vez que en nuestro país aún no se regula una ley de reproducción humana asistida, que contemple estas situaciones fácticas y proteja los derechos de estos niños, por considerar al Juez un creador del derecho a tenor de lo prescrito en el inciso 8 del Artículo $139^{\circ}$ de la Constitución Política del Estado. Todo ello, en observancia además de la Novena Parte del Preámbulo de la Convención sobre los Derechos del Niño, que establece "...Teniendo presente, como se indica en la Declaración de los Derechos del Niño, "el niño por su falta de madurez física y mental, necesita protección y cuidado especiales, incluso la debida protección legal, tanto antes como después del nacimiento"..." crecer, a nacer, son considerados derechos indisponibles.

Que tomando como base el derecho comparado, y en dicho caso concreto, la legislación española, la Ley 14/2006 sobre "Técnicas de Reproducción Asistida", del 26 de mayo del 2006, en su Artículo $11^{\circ}$ establece los diferentes destinos posibles que podrán darse a los embriones crio-conservados, como: 1) Su utilización por la propia mujer o su cónyuge; 2) La donación con fines reproductivos; 3) La donación con fines de investigación; y 4) El cese de su conservación sin otra utilización (la muerte de dicho embrión));

De acuerdo a nuestro derecho nacional, que recoge el Sistema de Protección Integral, solo resulta válido, ética y moralmente aceptable la primera alternativa, dado que las dos siguientes, al considerar al embrión, no sujeto de derecho, sino objeto de derecho, afecta principios elementales de la dignidad humana, dado que la persona es siempre un valor en sí y por sí misma y no puede ser tratada como objeto utilizable, como instrumento o medio, y por esta sustantiva razón se le debe respeto desde el primer instante de su existencia, más aún que conforme la última parte del Artículo $7^{\circ}$ de la Ley General de Salud, Ley Número 26842, "Está prohibida la fecundación de óvulos humanos con fines distintos a la procreación, así como la clonación de seres humanos."; La última alternativa, tampoco la consideramos adecuada, por cuanto implica la muerte de los embriones crio-conservados lo que conlleva a la vulneración del derecho que tiene todo ser humano a la vida desde su

\footnotetext{
${ }^{79}$ Expediente número 183515-2006-00113.

${ }^{80}$ Norma jurídica supranacional que forma parte de nuestro derecho interno, en mérito a los dispuesto en la Cuarta Disposición Final y Transitoria de la Constitución Política del Estado en concordancia con el Artículos $3^{\circ}$ de la misma norma legal;
} 
concepción; que por dichas razones se dictaron medidas destinadas a hacer efectivo el derecho a la vida que tiene todo concebido en su calidad de niño, de conformidad con lo dispuesto en el inciso $1^{\circ}$ del Artículo $2^{\circ}$ de la Constitución Política del Estado.

Que dichas medidas constituyeron en: 1) Otorgar el plazo de dos años a efectos que los padres de los "concebidos" hagan efectivo el derecho a la vida que tienen los citados embriones concebidos producto de la fecundación In Vitro de sus ovocitos y espermatozoides, que se encuentran vivos y congelados, sea mediante implantación en el vientre materno o una subrogación de vientre de tercera persona sin fines de lucro, contados a partir de que dicha resolución quede consentida y/o ejecutoriada; 2) Disponer que vencido dicho plazo, si los citados justiciables no cumplieran el precitado mandato, cursar oficios al Juzgado de Familia Tutelar respectivo o al Ministerio de la Mujer y Desarrollo Social, según sea el caso, a efectos de que inicie el proceso tutelar de abandono de los citados embriones congelados y puedan otorgarse en adopción a padres sustitutos, con la finalidad de hacer efectivo el "Derecho a la vida" que tienen aquellos seres humanos, en su calidad de niños y por ende Sujetos de Derechos y de Protección Específica; 3) También se dispuso que la Defensoría del Pueblo, en su calidad de "Amicus curiae" (amigo de la Corte) y encargado de la defensa de los derechos fundamentales de la persona humana, de acuerdo al artículo $162^{\circ}$ de la Constitución Política del Estado y artículo $1^{\circ}$ de su Ley Orgánica, ley número 26520, supervise el cumplimiento y la ejecución de dicha sentencia.

Que tales medidas se tomaron considerando en primer lugar que el derecho a la vida es un derecho fundamental que no se puede soslayar, que permitir lo contrario, aun por omisión, constituye un atentado contra la ética, que el Órgano Jurisdiccional del Estado no puede avalar; que el permitir que seres humanos permanezcan en estado de desarrollo humano de "concebido" y "congelados" en forma indeterminada, flagrante inobservancia a lo dispuesto en el artículo $6^{\circ}$ de la Convención sobre los Derechos del Niño que regula que: " 1 . Los Estados Partes reconocen que todo niño tiene el derecho intrínseco a la vida. 2. Los Estados Partes garantizarán en la máxima medida posible la supervivencia y el desarrollo del niño...", concordado con el artículo $3^{\circ}$ de dicha norma supranacional que prescribe: "1. En todas las medidas concernientes a los niños que tomen las instituciones públicas o privadas de bienestar social, los tribunales, las autoridades administrativas o los órganos legislativos, una consideración primordial a que se atenderá será el interés superior del niño. 2. Los Estados Partes se comprometen a asegurar al niño la protección y el cuidado que sean necesarios para su bienestar, teniendo en cuenta los derechos y deberes de sus padres,... y, con ese fin, tomarán todas las medidas legislativas y administrativas adecuadas.", que por lo tanto estando a que han sido los padres los que los han concebido utilizando dicha técnica, aquellos tienen el deber, en primer orden, de hacer efectivo tales derechos.

También se previno que si por razones de fuerza mayor aquellos no pudiesen hacer efectivo los derechos de los niños en el plazo de dos años, es el Juzgado de Familia o el Ministerio de la Mujer, quienes deben promover el proceso tutelar de abandono de los citados embriones congelados y pueda otorgarse en adopción a padres sustitutos, con la finalidad de hacer efectivo su "Derecho a la vida", toda vez que existen en la actualidad listas de espera de varios años de parejas que desean adoptar un niño, siendo que los "embriones sobrantes" deben descongelarse con el fin de transferirlos al útero de las madres adoptivas que desean gestarlos. Implantándose así el proceso de adopción prenatal, aplicándose supletoriamente las normas que regulan la adopción de nacidos (Adopción tutelar o excepcional), y así puede darse cumplimiento a los derechos de los niños, y de los adoptantes, esto es los derechos de 
vivir y procrear, respectivamente. Que para evitar que las disposiciones de dicha sentencia judicial queden en olvido, dado que un expediente concluido, necesariamente queda archivado a los cuatro meses por falta de impulso procesal, según normas administrativas de la Corte Superior de Justicia de Lima, se dispuso que se supervise el cumplimiento y la ejecución de dicha sentencia, por la Defensoría del Pueblo considerando su rol de protector, promotor y defensor de los derechos fundamentales;

Que dicha sentencia fue elevada en Consulta a la Corte Suprema de Justicia de la Republica, vía control difuso, en merito a lo dispuesto en el artículo 14 de la Ley Orgánica del Poder Judicial, por: 1) Haber aplicado el derecho que corresponde al proceso por vacío y deficiencia de la ley; y 2) Haber dispuesto de oficio, pese a no ser parte del petitorio, medidas tendientes a preservar y hacer efectivo el Derecho a la Vida de los tres embriones vivos que se encuentran congelados en la citada institución médica privada, por constituir derechos indisponibles, inaplicado las restricciones dispuestas en el artículo VII del Título Preliminar del Código Procesal Civil, respecto a la prohibición de fallo "Ultra y Extra Petita", al prevalecer el Principio y Derecho de la función jurisdiccional de "la observancia del debido proceso y tutela jurisdiccional efectiva de tres concebidos, indefensos, sujetos de protección y cuidados especiales" conforme lo prescribe el inciso 3 del artículo $138^{\circ}$ de la Constitución Política del Estado, en concordancia con el Noveno Preámbulo de la citada Convención sobre los Derechos del Niño.

La Sala de Derecho Constitucional y Social Permanente de la Corte Suprema de Justicia de la Republica desaprobó dicho extremo consultado, con la sola consideración "que se ha incurrido en vicio de congruencia extra - petita al no haber sido materia de la pretensión demandada"; que sin embargo, la Suprema Corte no consideró que tratándose de derechos indisponibles, en los que están involucrados derechos de niños, el Estado tiene el poder - deber de impartir medidas necesarias a efectos de preservar sus derechos, conforme lo regula el artículo $4^{\circ}$ de la Convención sobre los Derechos del Niño que prescribe que "Los Estados Partes adoptarán todas las medidas administrativas, legislativas y de otra índole para dar efectividad a los derechos reconocidos en la presente Convención." La que, como se repite, forma parte del derecho nacional. ${ }^{81}$. Que aún más, el Tercer Pleno Casatorio de la Corte Suprema Republica, Casación 4664-2010-Puno, emitido con fecha 18 de marzo del 2011, dejo establecido que "...las finalidades fundamentales tuitivas a la familia trascienden a los intereses estrictamente individuales, de modo que su incumplimiento no puede dejarse al arbitrio individual...".

${ }^{81}$ Que en efecto, así se lee en dicho precedente vinculante: “...Que el derecho procesal de familia se concibe como aquel destinado a solucionar con prontitud los conflictos que surjan dentro de la esfera de las relaciones familiares y personales ofreciendo protección a la parte perjudicada, ya sea que se trate de hijos, padres, cónyuges, hermanos, etc. De allí que se diferencie del proceso civil en razón de la naturaleza de los conflictos a tratar, y que imponen al Juez una conducta conciliadora y sensible, que supere los formalismos y las meras cuestiones técnicas....La naturaleza del derecho material de familia, en sus diversas áreas y en distintos grados condiciona al legislador y al Juez para regular y desarrollar procesos que correspondan a aquella naturaleza, evitando el exceso ritual y la ineficacia del instrumento procesal. Se comprende por ello que por un lado, el proceso tenga una estructura con componentes flexibles y, por otro tenga el Juez de Familia amplias facultades tuitivas, para hacer efectivos aquellos derechos. Las finalidades fundamentales tuitivas a la familia trascienden a los intereses estrictamente individuales, de modo que su incumplimiento no puede dejarse al arbitrio individual... Cabe preguntarnos si puede considerarse infracción al Principio de Congruencia Procesal cuando un Juez de Familia decide sobre pedidos o petitorios implícitos... en tal sentido no resulta lógico que, al encontrarnos frente a un proceso tuitivo, no pueda permitirse la flexibilización del Principio de Congruencia al interior del proceso para efectos de revisar y dar solución al conflicto en sí mismo, independientemente de la forma o términos en los que se hubiere planteado la demanda...". 
Que en ejercicio al derecho a la crítica de las resoluciones judiciales prevista constitucionalmente, dicha sentencia ha sido cuestionada académicamente ${ }^{82}$, en el sentido que "...el obligar a una mujer enferma a gestar por si o bien buscar quien geste por ella, a tres óvulos fecundados crio conservados, y poner a tal fin un plazo perentorio de dos años, bajo amenaza de iniciarle a ella y a su esposo un proceso de abandono de menores es ir demasiado lejos. ¿Dónde queda el derecho a elegir el número y espacio entre los hijos?, ¿Dónde su derecho a la integridad, a la libertad, al libre desarrollo de la personalidad? ¿De que manera se respeta su dignidad al convertirla a ella a riesgo de su vida, o bien a una tercera mujer, con el costo físico y emocional que una maternidad subrogada conlleva, en una incubadora?..."; que efectivamente nos encontramos frente a dos derechos constitucionales opuestos, nos referimos a los derechos de tres niños, seres humanos en estado de "concebidos", que no pueden valerse por sí mismos, cuyos derechos fundamentales se encuentran objetivamente vulnerados, y los derechos de dos personas (los padres) con plena capacidad de ejercicio, quienes decidieron voluntariamente vía fecundación in vitro procrear a estos niños, los mismos que se encuentran en estado de indefensión "congelados vivos", recordemos que un derecho constitucional, por su naturaleza no es absoluto, será mediante una ponderación de derechos, en el que se determinará cuál de ellos cede para dar prioridad al otro, por nuestra parte, está clara la decisión adoptada;

Que por otro lado, la disposición emitida en el sentido de que si en el plazo de dos años los padres no hacen efectivo el derecho a la vida de sus hijos, deben ser dados en adopción, a fin de transferirlos al útero de madres adoptivas que deseen gestarlos; ello de ninguna manera puede constituir una amenaza para los padres, muy por el contrario, constituye la respuesta por parte del Estado en otorgar a los padres el derecho de preferencia en hacer efectivo los derechos fundamentales de tales niños, como el derecho a la vida, el derecho a nacer, el derecho a crecer, y demás derechos inherentes a la persona humana, toda vez que de ninguna manera el Estado puede permitir o soslayar que "un niño", "un concebido" quede en la eternidad congelado, vivo y sin protección legal; lo contrario implica afectar derechos fundamentales que la ética ni el derecho permiten ; de allí la necesidad imperante de regular sobre esta materia.

${ }^{82}$ Por Paula Siverino Bavio, abogada por la Universidad de Buenos Aires. Profesora de Derecho Civil y Bioética de la Pontificia Universidad Católica del Perú e Inca Garcilaso de la Vega, en su Artículo "¿Quién llamo a la cigüeña? Maternidad Impugnada e Identidad Genética." Pub. En Dialogo con la Jurisprudencia, Tomo 141, Junio del 2010, Pág. 140/160. 


\section{CONCLUSIONES}

1. Según el artículo $1^{\circ}$ del código civil, la atribución de derechos patrimoniales al concebido, está condicionado a que nazca vivo. Norma que implícitamente ha quedado derogada por el código de los niños y adolescentes que define al niño como persona humana desde la concepción, (art. I, título preliminar), ello para evitar manipulaciones o experimentos genéticos.

2. En el Perú aún no se expide una ley de reproducción humana asistida que regule estas situaciones fácticas y proteja los derechos de estos niños por considerar al juez un creador del derecho a tenor de lo prescrito en el inciso h del artículo 139 de la constitución política del Estado.

3. Los diferentes destinos posibles que podrían darse a los embriones crio conservados su utilización, su donación o el cese de su conservación, (muerte de dicho embrión).

4. El derecho a la vida es un derecho fundamental que no se puede soslayar, por tanto, su omisión constituye un atentado contra la ética que no puede ser avalado por el órgano jurisdiccional del Estado.

5. Existen en la actualidad listas de espera, de varios años, de parejas que desean adoptar un niño; consecuentemente sería conveniente que los embriones sobrantes sean descongelados con el fin de transferirlos al útero de las madres adoptivas que deseen gestarlos; implantándose así el proceso de adopción pre natal, aplicándose supletoriamente las normas que regulan la adopción de nacidos y así poderse dar cumplimiento a los derechos de los niños y de los adoptantes, es decir vivir y procrear respectivamente.

6. El Estado no puede permitir que un niño, un concebido quede eternamente congelado vivo y sin protección legal. Lo contrario implica afectar derechos fundamentales que la ética ni el derecho permiten; de allí la imperiosa necesidad de legislar sobre la materia.

\section{BIBLIOGRAFIA}

- Siverino Bavio, Paulo. (2010). ¡Quién llamó a la cigüeña?. Maternidad impugnada e identidad genética. Diálogos con la jurisprudencia, tomo 141 (pp.140/160)

- Casación 4664-2010 del 18.03.11 Puno

- Código de los niños y adolescentes

- Código civil (art. 1)

- Convención sobre los derechos del niño. ONU ratificada por resolución legislativa 25278 del 03.08 .90

- Constitución política del Estado (4ta. Disposición final y transitoria)

- Expediente sobre fecundación in vitro $N^{\circ} 183515-2006-00113$

- Ley 26520 Ley orgánica de la defensoría del pueblo

- Ley general de salud

- Ley 14/2006. Técnicas de reproducción humana asistida. España 


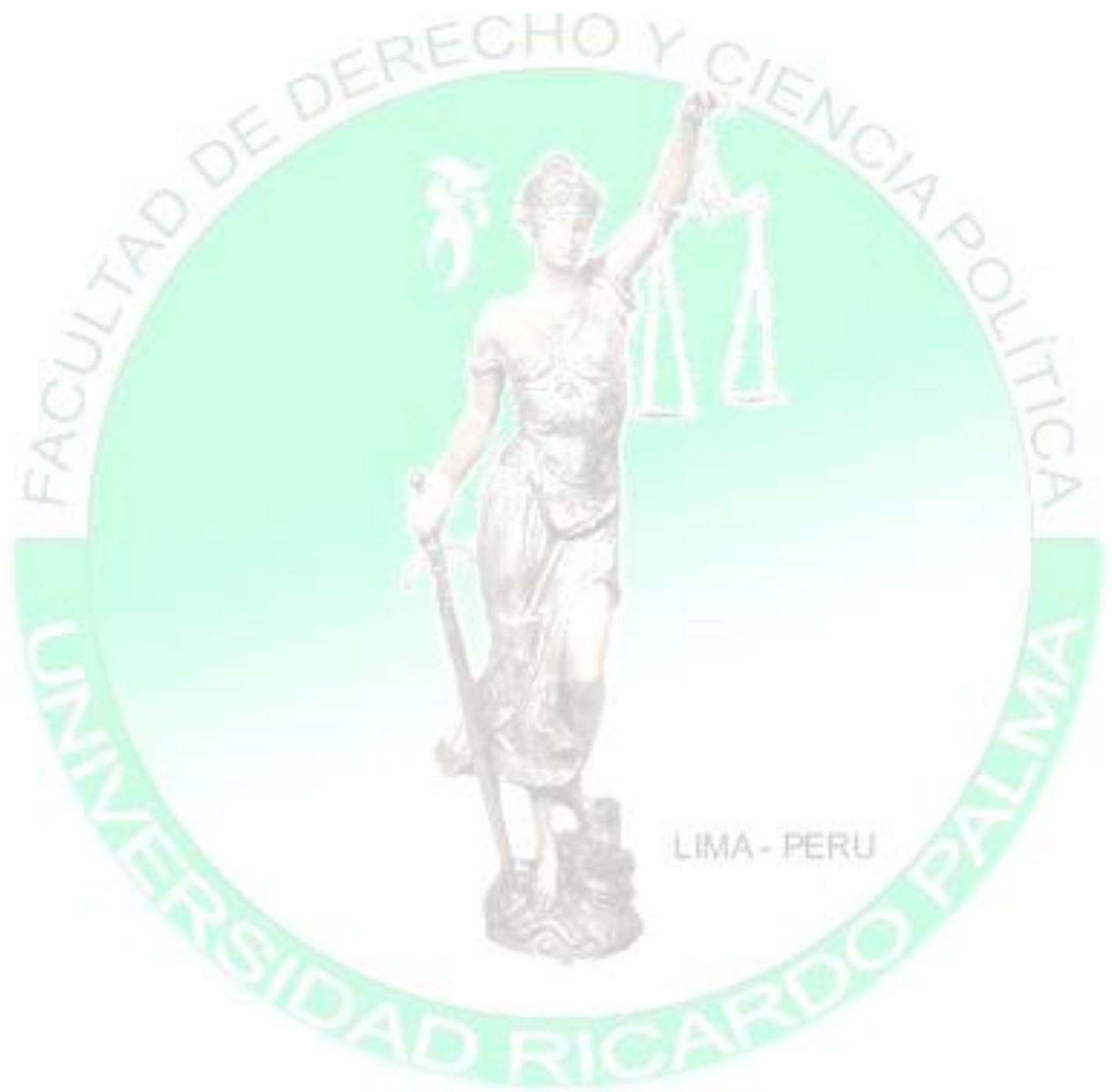

